\title{
Sequential Administration of Interleukin-6 and Granulocyte-Colony Stimulating Factor in Newborn Rats: Modulation of Newborn Granulopoiesis and Thrombopoiesis ${ }^{1}$
}

\author{
MITCHELL S. CAIRO, J. MICHAEL PLUNKETT, ANNA NGUYEN, \\ STEVEN CLARK, AND CARMÉLLA VAN DE VEN \\ Division of Hematology/Oncology, Children's Hospital of Orange County, Orange, California 92668 and \\ Genetics Institute, Cambridge, Massachusetts 02140
}

\begin{abstract}
During states of increased demand, neonatal host defense is characterized by dysregulation of granulopoiesis, resulting in a high incidence of neutropenia. This study investigated the modulation of neonatal rat hematopoiesis by 14-d administration of recombinant human (rh) IL-6, rh-granulocyte-colony stimulating factor (G-CSF), or sequential combination of rhIL-6 and rhG-CSF. Specifically, newborn Sprague-Dawley rats were treated with either rhIL-6 (5 $\mu \mathrm{g} / \mathrm{kg} / \mathrm{d}$ for $14 \mathrm{~d})$, rhG-CSF $(5 \mu \mathrm{g} / \mathrm{kg} / \mathrm{d}$ for $14 \mathrm{~d}$ ), rhIL-6 for $7 \mathrm{~d}$ followed by rhG-CSF for $7 \mathrm{~d}, \mathrm{PBS} /$ BSA for $7 \mathrm{~d}$ followed by rhG-CSF for $7 \mathrm{~d}$, or PBS/BSA for $14 \mathrm{~d}$. RhIL-6 alone significantly increased the peripheral platelet count during the latter part of the 2 nd wk of administration (d 13: $980 \pm 42$ versus $716 \pm 23 \times 10^{3}$ / $\left.\mathrm{mm}^{3}\right)(p=<0.001)($ mean \pm SEM). Treatment with rhIL6 for $7 \mathrm{~d}$ followed by rhG-CSF significantly increased the peripheral neutrophil count compared with $7 \mathrm{~d}$ of PBS/ BSA and $7 \mathrm{~d}$ of G-CSF (d 14 absolute neutrophil count $4888 \pm 12$ versus $\left.2720 \pm 317 / \mathrm{mm}^{3}\right)(p=<0.05)$. Similarly, sequential rhIL-6/rhG-CSF significantly increased the d14 bone marrow neutrophil storage pool $(9873 \pm 882$ versus $\left.3564 \pm 159 / \mathrm{mm}^{3}\right)(p=<0.005)$. Lastly, sequential rhIL6/rhG-CSF induced the highest increase in bone marrow $(p<0.01)$ and liver/spleen CFU-GM pool $(p<0.001)$ compared with any other treatment group. These studies suggest that rhIL-6 alone is associated with a significant increase in the neonatal platelet count. The sequential combination of rhIL-6 followed by rhG-CSF may also induce significant increases in neonatal peripheral neutrophilia, bone marrow neutrophil storage pools, and bone marrow and liver/spleen myeloid progenitor pools. This sequential combination has been demonstrated to enhance neonatal hematopoiesis. (Pediatr Res 30: 554-559, 1991)
\end{abstract}

\section{Abbreviations}

G-CSF, granulocyte-colony stimulating factor CFU-GM, colony forming unit granulocyte-macrophage CSF, colony-stimulating factor ANC, absolute neutrophil count rh, recombinant human

Received May 14, 1991; accepted July 15, 1991.

Correspondence: Mitchell S. Cairo, M.D., Director, Hematology/Oncology Research and Bone Marrow Transplantation, Children's Hospital of Orange County, 455 S. Main Street, Orange, CA 92668.

Supported by grants from the Pediatric Cancer Research Foundation, the Walden W. and Jean Young Shaw Foundation, and the CHOC Research and Education Foundation.

${ }^{\prime}$ Presented in part at the Society for Pediatric Research, April 1991, in New Orleans, LA.
HBSS, Hanks' balanced salt solution

Profound immaturities in neonatal hematopoiesis predispose the newborn to significant cytopenias. Specifically, during overwhelming bacterial sepsis, the newborn is susceptible to a high incidence of thrombocytopenia and neutropenia (1-5). Decreased total body neutrophil storage pools, reduced total body myeloid progenitor pools (CFU-GM), and maximal myeloid progenitor proliferative rates have been previously demonstrated in newborn rats versus adult animals (1-4).

Human IL-6, first purified to homogeneity from the HTLV II-transformed T cell line TCL/NA1, has been noted to stimulate pluripotent hematopoietic stem cells and to act synergistically with IL-3 in the stimulation of multilineage hematopoietic blast colonies (6-8). G-CSF, on the other hand, is a more myeloid lineage-specific CSF and has been demonstrated to stimulate myeloid proliferation and maturation and induce neutrophilia secondary to egress of neutrophil storage pool cells from bone marrow storage pool reserves (9-11).

IL-6 has recently been demonstrated to have significant in vitro effects on fetal myeloid progenitor proliferation (12). We have previously demonstrated that both single-pulse administration of rhG-CSF and a prolonged 7-d administration in newborn rats result in a significant increase in peripheral neutrophilia, modulation of myeloid proliferation, and reduction in morbidity after experimental group B streptococcal sepsis (13). In the present study, we investigated the modulation of in vivo neonatal hematopoiesis in the newborn rat by sequential administration of a nonlineage class I CSF such as IL-6 with a more lineagespecific class II CSF, G-CSF.

\section{MATERIALS AND METHODS}

$G$-CSF. RhG-CSF, kindly provided by Jeffrey Andresen, Amgen Laboratories, Thousand Oaks, CA, was prepared from an Escherichia coli host by recombinant DNA techniques. RhGCSF was prepared to $>95 \%$ purity before formulation in $0.025 \%$ human serum albumin. SDS-PAGE was used to confirm the purity of the growth factor by the appearance of a single protein band. Biologic activity of $2 \times 10^{8}$ units/mg was determined by granulocyte colony formation (CFU-G) on human nonadherent bone marrow cells in semisolid media. The Limulus amebocyte lysate assay (Sigma Chemical Co., St. Louis, MO) was used to demonstrate the absence of measurable endotoxin. The lower limits of detection of endotoxin using this assay were $0.05-0.1$ endotoxin units/mL. Purified G-CSF was used at a dose of $5 \mu \mathrm{g} /$ $\mathrm{kg} / \mathrm{d}$ by dilution with PBS, pH 7.4. 
RhIL-6. RhIL-6, kindly provided by Steven Clark, Genetics Institute, was expressed and cloned in $E$. Coli and purified to apparent homogeneity before lyophilization. Purity was greater than $99 \%$ by HPLC and by the presence of a single protein band by SDS-PAGE. Biologic activity of $4 \times 10^{9}$ units $/ \mathrm{mg}$ was determined by tritiated thymidine incorporation of $B 9$ hybridoma cells. Absence of measurable endotoxin was confirmed by the Limulus amebocyte lysate assay. RhIL- 6 was diluted in sterile PBS containing $0.025 \%$ BSA and used at a dose of $5 \mu \mathrm{g} / \mathrm{kg} / \mathrm{d}$.

Animal inoculation. Litters of albino Sprague-Dawley neonatal rats (Benton-Kingman Laboratories, Fremont, CA) $\leq 24 \mathrm{~h}$ old $(6-8 \mathrm{~g})$ were used during this study. Mothers of the litters were received $1 \mathrm{wk}$ before delivery and were housed at the vivarium at the University of California Irvine Medical Center. They were maintained at constant room temperature, with water and rodent feed (Purina Chow; Ralston-Purina Co., St. Louis, MO) ad libitum. Approval for this study was granted from the Animal Use Committee of the University of California Irvine.

Before the administration of growth factor, the site of injection was washed with Betadine solution (povidone-iodine, 10\%; Purdue Frederick, Norwalk, CT) and swabbed with $70 \%$ isopropol

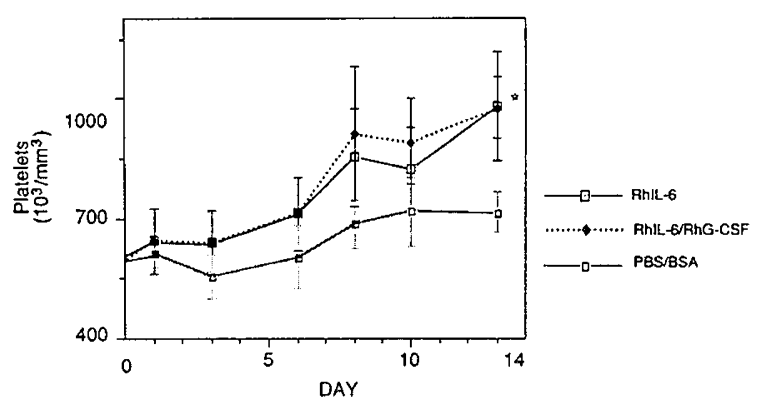

Fig. 1. Sprague-Dawley neonatal rats ( $\leq 24 \mathrm{~h}$ old $)$ received rhIL-6 daily for $14 \mathrm{~d}$ or rhIL-6 daily for $7 \mathrm{~d}$ followed by rhG-CSF daily for $7 \mathrm{~d}$; control animals received PBS/BSA daily for $14 \mathrm{~d}$. All treatments were administered by intraperitoneal injection. Blood samples were taken on $\mathrm{d} 1,3,6,8,10$, and 13, and electronic platelet counts were performed. Values presented are the mean \pm SEM of three experiments in which three to five animals were evaluated at each time point for each treatment group. *, RhIL-6 and rhIL-6/RhG-CSF versus PBS/BSA, d 13 ( $p<$ $0.001)$. alcohol. Intraperitoneal injections were accomplished with a sterile tuberculin syringe fitted with a 27.5-gauge needle. Animals were injected daily for $14 \mathrm{~d}$ with rhIL-6 $(5 \mu \mathrm{g} / \mathrm{kg})$ or rhG-CSF $(5$ $\mu \mathrm{g} / \mathrm{kg}$ ), or combinations of $7 \mathrm{~d}$ of rhIL- 6 followed by $7 \mathrm{~d}$ of $\mathrm{rhG}-$ CSF or $7 \mathrm{~d}$ of PBS/BSA and $7 \mathrm{~d}$ of rhG-CSF. Control animals received injections of $0.025 \%$ BSA in PBS daily for $14 \mathrm{~d}$.

Quantification of circulating and storage neutrophils. Blood samples were obtained by nicking the jugular vein or foot pad with a sterile needle and collecting $10 \mu \mathrm{L}$ of free flowing blood. Total blood cell counts including platelets and hematocrit levels were electronically measured (Serano-Baker Diagnostics, Allentown, PA). Blood smears were prepared and stained with Wright stain, and a 100-200 cell differential was performed. Absolute neutrophil counts were determined by the multiplication of the nucleated cell count by the percentage of neutrophils in the differentials. Neutrophil bone marrow pools were determined by the method of Christensen et al. (4). Briefly, neonate femurs were aseptically removed on $\mathrm{d} 14$ and the contents were flushed into a known quantity of HBSS (Gibco Laboratories, Grand Island, NY). Livers and spleens were also removed on d 14 in a similar manner and finely minced in a known quantity of HBSS. Electronic cell counts were performed on the bone marrow, and a 500-cell differential count was obtained on Wright-stained cytospin preparations.

$C F U-G M$ concentration. Bone marrow and liver/spleen cells were collected as described above. Cells were then suspended in alpha media (Gibco Laboratories) with $1.1 \%$ methylcellulose (Terry Fox Laboratories, Vancouver, BC), 30\% vol/vol FCS (Hyclone Laboratories, Logan, UT), 10\% BSA (Sigma Chemical Co.), $14.3 \times 10^{-3} \mathrm{M}$ mercaptoethanol, $0.01 \% \mathrm{vol} / \mathrm{vol}$ murine spleen cell conditioned medium (PWM-SCCM; Terry Fox Laboratories), and $1 \mathrm{U} / \mathrm{mL}$ erythropoietin (Amgen, Thousand Oaks, CA). Penicillin $(100000 \mathrm{U} / \mathrm{L})$ and streptomycin $(100 \mathrm{mg} / \mathrm{L})$ were also added. Cell suspensions were plated in triplicate in 10$\times 35$-mm tissue culture dishes (Nunc, Roskilde, Denmark) and incubated at $5 \% \mathrm{CO}_{2}, 37^{\circ} \mathrm{C}$, in a high humidity atmosphere. Cultures were evaluated at $14 \mathrm{~d}$ with aggregates of $>50$ cells considered "colonies." Colonies were plucked at random, placed on a slide, and stained, and lineage specificity was confirmed.

$C F U$ proliferative rate. Proliferative rates of CFU-GM were evaluated by the thymidine suicide method of Christensen $e t$ al. (4). Bone marrow and liver and spleen cells from neonatal rats were placed into each of two 50 -mL centrifuge tubes. To the first

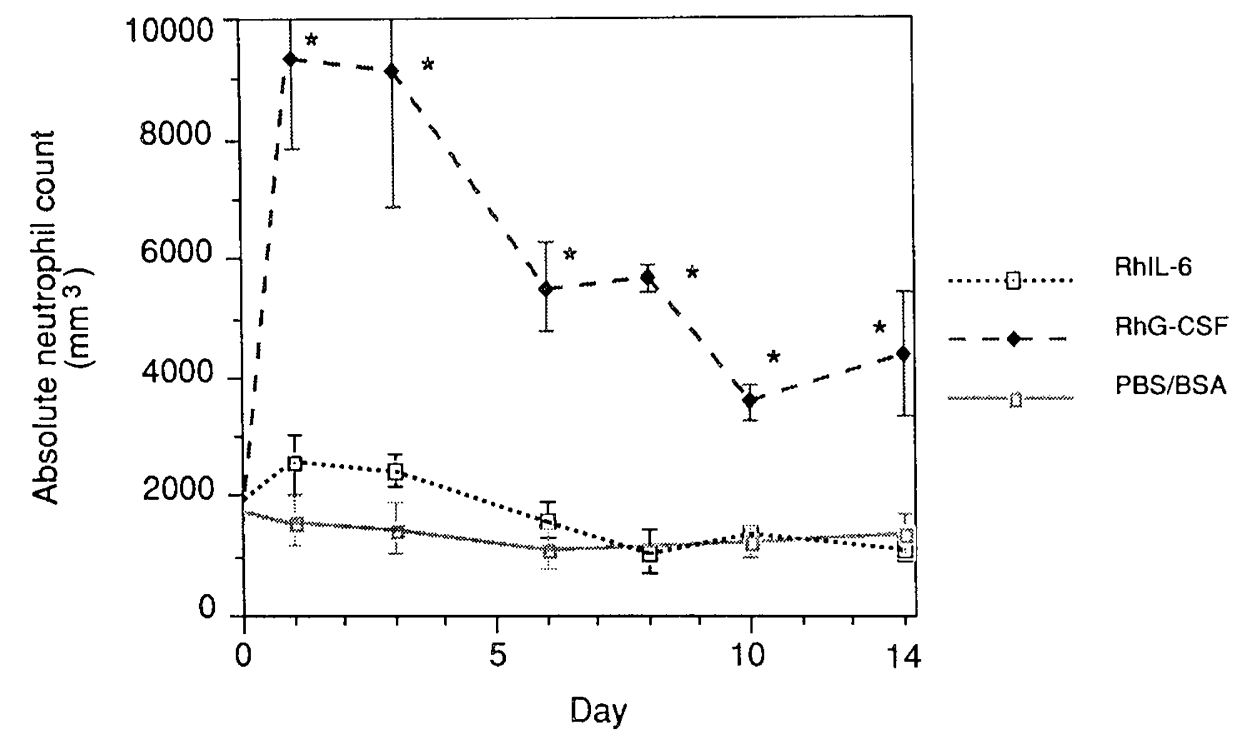

Fig. 2. Neonatal Sprague-Dawley rats $(\leq 24 \mathrm{~h}$ old) received rhG-CSF, rhIL-6, or PBS/BSA by intraperitoneal injection daily for $14 \mathrm{~d}$. Blood samples were taken on $\mathrm{d} 1,3,6,8,10$, and 13 and were electronically counted, and a 100 to 200-cell differential was performed on Wright's stained blood smears. Values presented are the mean \pm SEM of three experiments in which three to five animals were evaluated at each time point for each treatment group. *, RhG-CSF versus rhIL-6 and PBS/BSA $(p<0.001)$. 
aliquot was added $0.34 \mu \mathrm{g}$ nonradioactive thymidine in a volume of $0.1 \mathrm{~mL}$. The other aliquot received $0.34 \mu \mathrm{g}$ methyl- ${ }^{3} \mathrm{H}$-thymidine containing $0.1 \mathrm{mCi}$ (sp act $75 \mathrm{Ci} / \mathrm{mmol}$; ICN Radiochemicals, Irvine, CA). The tubes were incubated for $20 \mathrm{~min}$ at $37^{\circ} \mathrm{C}$ and were agitated every $5 \mathrm{~min}$. Thymidine uptake was halted by adding $30 \mathrm{~mL}$ of ice cold $\alpha$-minimum essential medium with $5 \%$ FCS and $100 \mu \mathrm{g} / \mathrm{mL}$ nonradioactive thymidine. The cell suspensions were then centrifuged and washed twice with the $\alpha$ minimum essential medium/FCS/thymidine media. Cells were then added in the same $1.1 \%$ methylcellulose/alpha media described above. Colonies were allowed to develop for $14 \mathrm{~d}$ in a $5 \% \mathrm{CO}_{2}$ incubator at $37^{\circ} \mathrm{C}$, and thymidine suicide rate was determined by subtracting the average number of colonies formed per plate by cells exposed to ${ }^{3} \mathrm{H}$-thymidine from the average number of colonies per plate formed by cells exposed to nonradioactive thymidine and dividing by the average colonies per plate from cells exposed to nonradioactive thymidine.

Statistical analysis. All results are expressed as mean values \pm SEM of eight to 15 animals, or three to five replicates of blood or bone marrow samples. The probability of significant differences when comparing two treated groups was determined with the use of the unpaired $t$ test, and the probability of significant differences when examining multiple treatments was determined by using the analysis of variance followed by the Student-Newman-Keuls multiple range tests to define the unique subsets within the study. Statistical analyses were performed using the Biostat I statistical program (Sigma Soft, Placentia, CA) for the IBM personal computer. $p$ values $<0.05$ are considered significant.

\section{RESULTS}

Peripheral blood counts including hematocrit, white cell count, differential, and platelet count were determined on d 1, 3, 6, 8, 10 , and 13 after intraperitoneal administration of rhIL-6 alone, rhIL- 6 and rhG-CSF, or PBS/BSA control. During the 1 st wk of administration, there was no significant difference in the peripheral platelet count between treatment groups. However, during the 2 nd wk of administration, it became apparent that rhIL-6 significantly increased the peripheral platelet count over that of control animals (PBS/BSA) (Fig. 1). The d-13 platelet count in the rhIL-6-treated animals was $980 \pm 42 \times 10^{3}$ versus $716 \pm 23$ $\times 10^{3} \mathrm{~mm}^{3}$ in the control animals $(p<0.001)$. There was, however, no significant enhancement in those animals pretreated for $7 \mathrm{~d}$ with rhIL-6 followed by $7 \mathrm{~d}$ of rhG-CSF therapy versus
$14 \mathrm{~d}$ of rhIL-6 alone (Fig. 1). Fourteen d of rhG-CSF had no effect on the platelet count (data not shown).

There was a mild decrease in the circulating hematocrit in all groups of treated animals, although there was no statistical difference between the rhIL-6, rhG-CSF, or control group animals [hematocrit: (d 7 rhIL-6, $29.3 \pm 0.6 \%$; rhG-CSF, $27.9 \pm$ $0.8 \%$; PBS/BSA, $27.5 \pm 0.8 \%$; d 14 rhIL-6, $25.6 \pm 0.3 \%$; rhGCSF, $26.0 \pm 0.6 \%$; PBS/BSA, $25.9 \pm 0.7 \%)(p=\mathrm{NS})]$. Additionally, there was no evidence of hemolysis on the peripheral smears from any of the treatment groups.

We also sought to determine whether there were any differences in the myeloid response between the different cytokine treatment arms. The absolute neutrophil count was calculated in each of the treatment groups. There was a significant difference between single and sequential cytokine therapy. In the single cytokine therapy, rhIL-6 alone failed to induce any significant increase in the circulating neutrophil count during the $14 \mathrm{~d}$ of administration compared with controls (PBS/BSA) (Fig. 2). RhG-CSF, however, as expected, induced a pronounced neutrophilia within $1 \mathrm{~d}$ of administration [d 1:9329 \pm 676 versus 1596 $\pm 178 / \mathrm{mm}^{3}$ (rhG-CSF versus PBS/BSA)] $(p<0.001)$ and continued to demonstrate a sustained neutrophilia throughout the $14 \mathrm{~d}$ of administration compared with control animals (Fig. 2) [d 14: $4400 \pm 609$ versus $1368 \pm 197 / \mathrm{mm}^{3}$ (rhG-CSF versus PBS/BSA $)](p<0.01)$. In the sequential cytokine treatment with rhIL- 6 for $7 \mathrm{~d}$ before $7 \mathrm{~d}$ of rhG-CSF therapy compared with PBS/BSA for $7 \mathrm{~d}$ before $7 \mathrm{~d}$ of G-CSF, there was a significant increase in the peripheral neutrophil count in the IL-6/G-CSF arm compared with PBS/BSA [d 14 ANC: $4888 \pm 12$ versus $2720 \pm 317 / \mathrm{mm}^{3}$ (rhIL-6/rhG-CSF versus $\mathrm{PBS} / \mathrm{BSA} / \mathrm{rhG}-\mathrm{CSF}$ )] $(p<0.05)$ (Fig. 3). Treatment with rhIL-6 for $7 \mathrm{~d}$ followed by rhG-CSF for $7 \mathrm{~d}$, however, did not significantly increase the peripheral ANC compared with $14 \mathrm{~d}$ of single-agent rhG-CSF therapy [d 14 ANC: $4888 \pm 17$ versus $4400 \pm 609 \mathrm{~mm}^{3}$ (rhIL$6 /$ rhG-CSF versus rhG-CSF)] ( $p=$ NS) (Figs. 2 and 3).

On d 14 of this experimental study, animals were killed, and neutrophil storage and proliferative pools were determined from aseptically surgically removed femurs, livers, and spleens. There was a significant increase in the bone marrow neutrophil storage pool (polymorphonuclear cells + band + metamyelocytes) in the animals pretreated with rhIL- 6 for $7 \mathrm{~d}$ followed by $7 \mathrm{~d}$ of rhGCSF compared with animals pretreated with control PBS/BSA for $7 \mathrm{~d}$ followed by rhG-CSF $(p<0.005)$ (Fig. 4). However, 14 $\mathrm{d}$ of single-agent rhG-CSF therapy induced the most significant increase in the bone marrow neutrophil storage pool $(p<0.002)$. Single-agent therapy with only rhIL- 6 failed to induce a signifi-

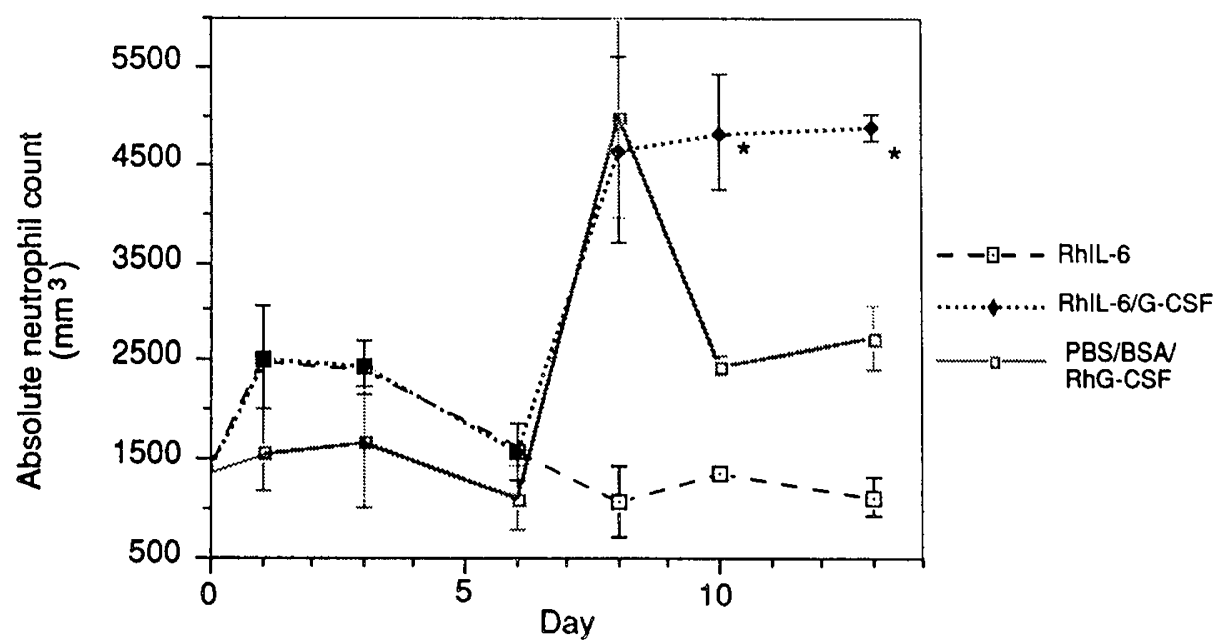

Fig. 3. Neonatal rats ( $\leq 24$ h old) received intraperitoneal injections of rhIL-6 for $14 \mathrm{~d}$, rhIL-6 for $7 \mathrm{~d}$ followed by rhG-CSF for $7 \mathrm{~d}$, or PBS/BSA for $7 \mathrm{~d}$ followed by rhG-CSF for $7 \mathrm{~d}$. Blood samples were taken on d 1, 3, 6, 8, 10, and 13 and were electronically counted, and a 100 to 200 -cell differential was performed on Wright's stained blood smears. Values presented are the mean \pm SEM of three experiments in which three to five animals were evaluated at each time point for each treatment group. *, RhIL-6/rhG-CSF versus $\mathrm{PBS} / \mathrm{BSA} / \mathrm{rhG}-\mathrm{CSF}(p<0.05)$. 


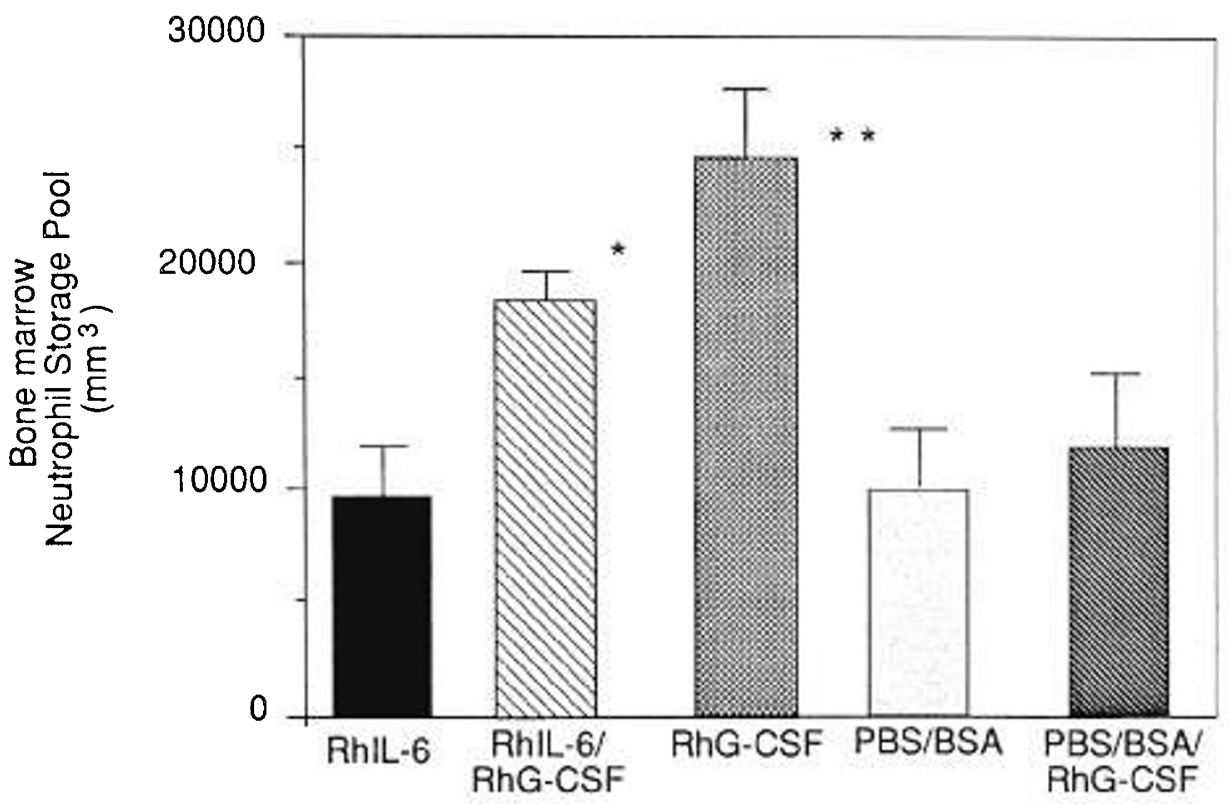

Fig. 4. Sprague-Dawley neonatal rats ( $\leq 24 \mathrm{~h}$ old) received the following cytokines by intraperitoneal injection: rhG-CSF, rhIL-6, or PBS/BSA daily for $14 \mathrm{~d}$, rhlL-6 daily for $7 \mathrm{~d}$ followed by rhG-CSF daily for $7 \mathrm{~d}$, or PBS/BSA daily for $7 \mathrm{~d}$ followed by rhG-CSF daily for $7 \mathrm{~d}$. Bone marrow neutrophil storage pool (polymorphonuclear cells + bands + metamyelocytes) was determined by removing the femurs and flushing the bone marrow into a known quantity of HBSS. Electronic cell counts were performed, and a 500-cell differential was obtained on Wright's stained cytospin preparations. Bars reflect the mean \pm SEM of three experiments of triplicate cultures of pooled samples of eight to 10 animals per treatment group. *, RhIL-6/rhG-CSF versus PBS/BSA/rhG-CSF $(p<0.005)$; ${ }^{* *}$, rhG-CSF versus PBS/BSA/rhG-CSF or PBS/BSA $(p<0.002)$.

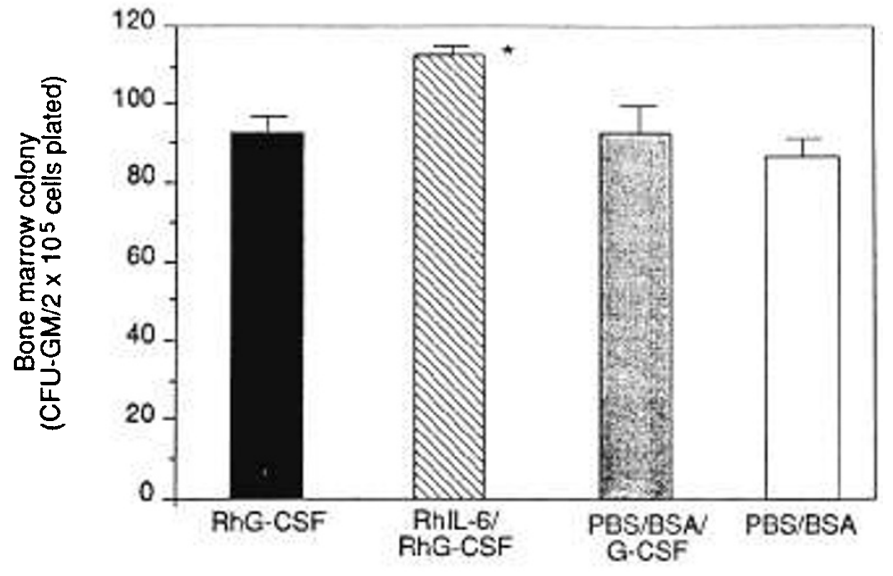

Fig. 5. Sprague-Dawley neonatal rats ( $\leq 24 \mathrm{~h}$ old) received rhG-CSF daily for $14 \mathrm{~d}$, rhIL-6 daily for $7 \mathrm{~d}$ followed by rhG-CSF daily for $7 \mathrm{~d}$, PBS/BSA daily for $7 \mathrm{~d}$ followed by rhG-CSF daily for $7 \mathrm{~d}$, or PBS/BSA daily for $14 \mathrm{~d}$. All treatments were administered by intraperitoneal injection. On $\mathrm{d} 14$ the animals were killed, their femurs were removed, and their bone marrow was flushed into HBSS. Cells $\left(10^{5}\right)$ were suspended in the growth media described, plated in triplicate dishes, and incubated at $5 \% \mathrm{CO}_{2}, 37^{\circ} \mathrm{C}$, at high humidity. CFU-GM colonies were evaluated at $14 \mathrm{~d}$, and bars represent the mean \pm SEM of three experiments of triplicate cultures of pooled samples of eight to 10 animals per treatment group. *, RhIL-6/rhG-CSF versus rhG-CSF $(p<0.01)$.

cant increase in the bone marrow neutrophil storage pool compared with control animals treated with PBS/BSA alone (Fig. 4). The neutrophil proliferative pool (blasts + promyelocytes + myelocytes), however, was not significantly different between treatment groups.

Bone marrow myeloid proliferative pools (CFU-GM) were also determined on $\mathrm{d} 14$ after $14 \mathrm{~d}$ of cytokine administration. $\mathrm{RhG}-\mathrm{CSF}$ induced a similar increase in the bone marrow CFU. GM as PBS/BSA/G-CSF and PBS/BSA alone. The sequential combination of rhIL- 6 for $7 \mathrm{~d}$ followed by rhG-CSF had a significant additive effect over that of any of the other treatment arms $(p<0.01)$ (Fig. 5). RhIL-6 alone had no significant effect on CFU-GM production. Additionally, sequential therapy of 7 $\mathrm{d}$ of rhIL- 6 followed by $7 \mathrm{~d}$ of rhG-CSF significantly increased the liver/spleen CFU-GM pools compared with $14 \mathrm{~d}$ of rhG-CSF and the other treatment groups $(p<0.008)$ (Fig. 6). CFU-GM proliferative rates were also determined from the bone marrow and liver and spleen CFU-GM colonies. There was, however, no significant difference in the CFU-GM proliferative rate among cytokine-treated groups.

\section{DISCUSSION}

Newborns are unusually susceptible to bacterial sepsis because of a profound immaturity in both quantitative and qualitative phagocytic immunity. Peripheral neutropenia is, in fact, a hallmark finding in overwhelming bacterial sepsis in the human newborn and has traditionally been associated with a poor outcome $(14,15)$. Future optimal therapy of neonatal sepsis or optimal prophylaxis may require adjuvant immunologic therapy to maintain adequate circulating numbers and qualitatively normal mature effector neutrophils.

IL-6 appears to act as a class I CSF, increasing early pluripotent blast cell colony formation with very little effect on mature progenitor proliferation (16). IL-6 has additionally been demonstrated to synergistically increase pluripotent bone marrow progenitor cells with IL-3 $(8,16)$. IL-6 and IL-3 also appear to synergistically stimulate purified human MY10 (CD34 $4^{+}$bone marrow progenitor cells (17). The synergism of IL-6 and IL-3 suggests that IL- 6 may be acting on the induction of noncycling (G0) progenitor cells and may induce maturation into more committed lineage-specific progenitor activity. In vitro stimulation with rhIL- 6 and cord peripheral blood and adult marrow progenitor cells suggests that rhIL-6 may support clonogenic maturation of multilineages, including CFU-GM, burst forming unit-erythorid, and colony forming unit-mixed colonies (12).

G-CSF is a more lineage-specific CSF. In serum-free cultures, G-CSF induces CFU-G formation but, in the presence of accessory cells or serum, may additionally support the formation of 


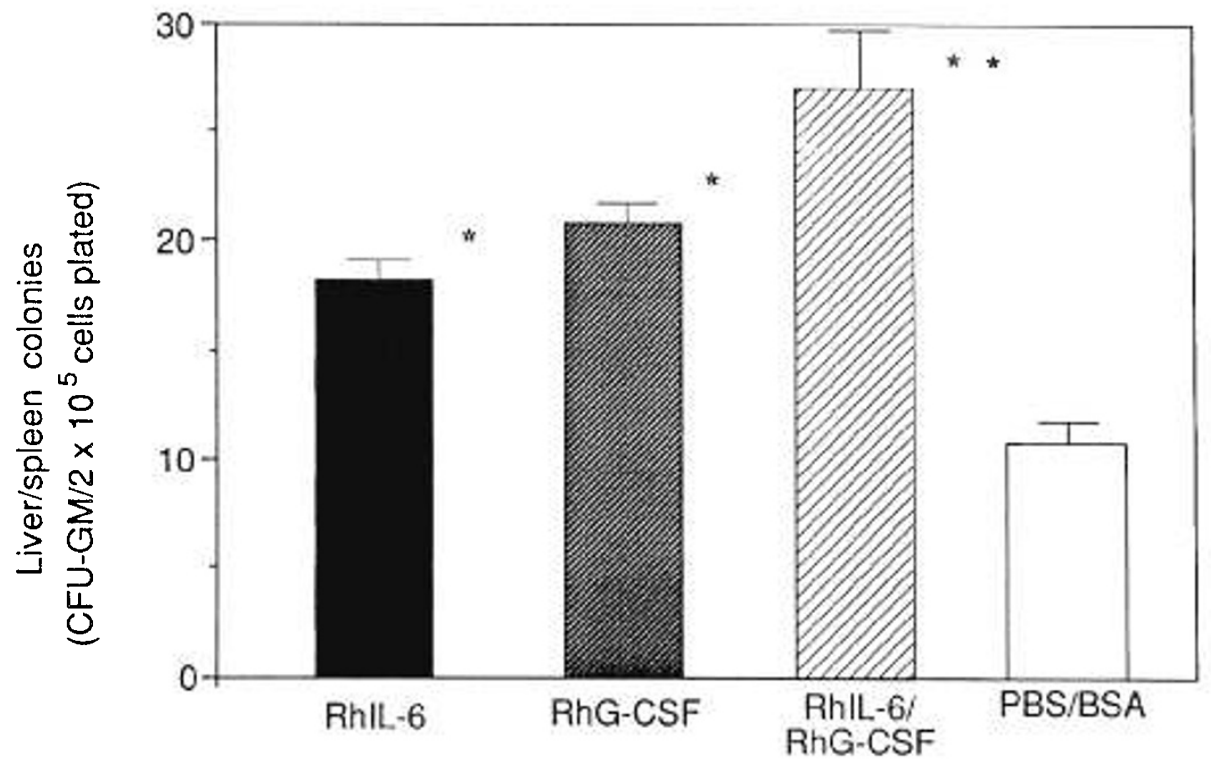

Fig. 6. Sprague-Dawley neonatal rats ( $\leq 24 \mathrm{~h}$ ) received rhIL-6 daily for $14 \mathrm{~d}$, rhG-CSF daily for $14 \mathrm{~d}$, rhIL-6 daily for $7 \mathrm{~d}$ followed by rhG-CSF daily for $7 \mathrm{~d}$, or PBS/BSA daily for $14 \mathrm{~d}$. All treatments were administered by intraperitoneal injection. On d 14 the animals were killed and liver and spleen were removed and reduced to a single cell suspension. Cells $\left(10^{5}\right)$ were suspended in the growth media described, plated in triplicate dishes, and incubated at $5 \% \mathrm{CO}_{2}, 37^{\circ} \mathrm{C}$, at high humidity. CFU-GM colonies were evaluated at $14 \mathrm{~d}$, and bars represent the mean \pm SEM of three experiments of triplicate cultures of pooled samples of eight to 10 animals per treatment group. ${ }^{*}$ RhIL- 6 and rhG-CSF versus PBS/BSA $(p<0.001)$, and ${ }^{* *}$, rhIL-6/rhG-CSF versus rhG-CSF $(p<0.008)$.

other lineages $(18,19)$. We have demonstrated that prolonged administration of rhG-CSF in newborn Sprague-Dawley rats results in a significant increase in the peripheral neutrophil count, the $\mathrm{d}-7$ bone marrow neutrophil storage pool, and the $\mathrm{d}-7$ bone marrow myeloid CFU-GM progenitor pool and significantly modulates the mortality rate after experimental group B streptococcal infection (20).

In the present study, it appears that administration of rhIL-6 induced a significant increase in the peripheral platelet count during the 2 nd wk of therapy compared with control animals. In previous adult animal studies, rhIL- 6 has been demonstrated to possess potent thrombopoietic activity when administered on consecutive days to adult animals (21-23). Similar increases in the platelet count during the $2 \mathrm{nd}$ wk of therapy have been demonstrated in cynomolgus monkeys receiving 14 consecutive $\mathrm{d}$ of rhlL-6 $(5-80 \mu \mathrm{g} / \mathrm{kg} / \mathrm{d})(21)$. The increase in the peripheral neutrophil count after 14 consecutive $d$ of rhG-CSF therapy in neonatal animals is consistent with our previous 7-d neonatal animal trial (20) and with adult animal trials by Ulich et al. (24). Fourteen d of rhG-CSF therapy to neonatal Sprague-Dawley rats also resulted in a very significant increase in the bone marrow neutrophil storage pool, which is consistent with the findings of our previous 7-d newborn rat studies (20). RhIL-6 alone, however, failed to induce a significant increase in the peripheral neutrophil count and the $\mathrm{d}-14$ bone marrow neutrophil storage pool. Single rhIL-6 cytokine therapy in neonatal animals produced a myeloid response similar to that which has been noted in adult animals $(21-23)$.

It is noteworthy, however, that combination sequential therapy of $7 \mathrm{~d}$ of rhIL- 6 followed by $7 \mathrm{~d}$ of rhG-CSF induced a significant increase in the circulating absolute peripheral neutrophil count in the 2 nd wk of therapy compared with $7 \mathrm{~d}$ of pretreatment with PBS/BSA. Combination sequential therapy of rhIL-6 followed by rhG-CSF also induced a significant increase in the d14 bone marrow neutrophil storage pool compared with PBS/ BSA followed by rhG-CSF. Additionally, and most importantly, it appeared that rhIL-6 therapy for $7 \mathrm{~d}$ followed by rhG-CSF induced the largest increase in the bone marrow and liver/spleen CFU-GM pools compared with any other combination of cytokine therapy. Thus, the number of myeloid progenitors in the bone marrow and liver/spleen appear to be the highest in the sequential rhIL-6/rhG-CSF group compared with rhG-CSF or rhIL-6 alone. This increase in bone marrow and liver/spleen myeloid progenitor pool may be the result of using a nonlineagespecific CSF such as IL-6 before a more myeloid lineage-specific CSF, such as G-CSF. The clinical relevance to this increased myeloid progenitor pool with this sequential combination still remains to be determined.

Previous studies using sequential therapy with an early-acting less lineage-specific cytokine (IL-3) followed by a more lineagespecific myeloid cytokine (GM-CSF) have been performed in other adult animal trials $(25,26)$. This sequential combination induced an increase in the leukocyte, platelet, and reticulocyte counts and a significant increase in the peripheral blood CFUGM pool (63-fold) compared with CFU-GM levels with rhIL-3 (14-fold) or rhGM-CSF (12-fold) alone $(25,26)$.

In summary, we have demonstrated that $14 \mathrm{~d}$ of rhIL-6 therapy to newborn Sprague-Dawley rats results in a significant increase in the peripheral platelet count during the latter part of the 2 nd wk of therapy, but has minimal effect on the myeloid response (peripheral neutrophilia, bone marrow neutrophil storage pool, and bone marrow CFU-GM). Fourteen $d$ of rhG-CSF therapy, however, induced the greatest increase in the peripheral neutrophil count and peripheral bone marrow neutrophil storage pool. RhG-CSF therapy had minimal effect on the circulating peripheral platelet count. The sequential combination of $7 \mathrm{~d}$ of rhIL-6 followed by $7 \mathrm{~d}$ of $\mathrm{rhG}$-CSF therapy, however, induced the highest increase in the bone marrow and liver/spleen CFU-GM pools compared with other cytokine-treated groups. This sequential combination additionally increased the peripheral neutrophil count and bone marrow storage pool compared with PBS/BSA/ rhG-CSF therapy. These studies suggest that sequential therapy of rhIL- 6 and rhG-CSF may be advantageous in inducing the highest number of myeloid bone marrow and liver/spleen progenitor cells, may maintain a reasonably high circulating peripheral neutrophil count and bone marrow neutrophil storage pool, and may promote an increase in the platelet count.

Acknowledgment. The authors thank Linda Rahl for her expert editorial assistance in the preparation of this manuscript. 


\section{REFERENCES}

Cairo MS 1989 Neonatal neutrophil host defense. Am J Dis Child 143:40-46

2. Christensen R, Rothstein G 1984 Pre and post-natal development of granulocyte stem cells (CFUc) in the rat. Pediatr Res 18:599-602

3. Erdman SH, Christensen RD, Bradley PP, Rothstein G 1982 Supply and release of storage neutrophils: a developmental study. Biol Neonate 41:132137

4. Christensen R, Shigeoka A, Hill H, Rothstein G 1980 Circulating and storage neutrophil changes in experimental type Il group B streptococcal sepsis. Pediatr Res 14:806-808

5. Cairo M, Worcester C, Rucker R, Bennetts G, Amlie R, Perkin R, Anas N, Hicks D 1987 Role of circulating complement and polymorphonuclear leukocyte transfusion in treatment and outcome in critically ill neonates with sepsis. J Pediatr 110:935-941

6. Kishimoto T 1989 The biology of interleukin-6. Blood 74:1-10

7. Hirano T Yasukawa K Harada H, Taga T Watanabe Y, Matsuda T, Kashiwaramura S, Nakajima K, Koyama K, Iwamatsu A, Tsunasawa S, Sakiyama F, Matsui H, Takahara Y, Taniguchi T, Kishimoto T 1986 Complementary DNA for a novel human interleukin (BSF-2) that induces B lymphocytes to produce immunoglobulin. Nature 324:73-76

8. Ikebuchi K, Wong G, Clark S, Ihle J, Hirai Y, Ogawa M 1987 Interleukin-6 enhancement of interleukin-3-dependent proliferation of multipotential hemopoietic progenitors. Proc Natl Acad Sci USA 84:9035-9039

9. Welte K, Platzer E, Lu L, Gabrilove J, Levi E, Mertelsmann R, Moore M 1987 Purification and biochemical characterization of human pluripotent hematopoietic colony-stimulating factor. Proc Natl Acad Sci USA 82:1526-1530

10. Welte K, Bonilla M, Gillio A, Boone T, Potter G, Gabrilove J, Moore M, O'Reilly R, Souza L 1987 Recombinant human granulocyte colony-stimulating factor effects on hematopoiesis in normal and cyclophosphamidetreated primates. J Exp Med 165:941-948

11. Ulich T, Del Castillo J, Souza L 1988 Kinetics and mechanisms of recombinan human granulocyte-colony stimulating factor-induced neutrophilia. Am J Pathol 133:630-638

12. Gardner J, Liechty K, Christensen R 1990 Effects of interleukin-6 on fetal hematopoietic progenitors. Blood 75:2150-2155

13. Cairo M, Mauss D, Kommareddy S, Norris K, van de Ven C, Modanlou H 1990 Prophylactic or simultaneous administration of recombinant human granulocyte colony stimulating factor in the treatment of group B streptococcal sepsis in neonatal rats. Pediatr Res 27:612-616

14. Akenzua G, Hui Y, Milner R, Zipursky A 1974 Neutrophil and band counts in the diagnosis of neonatal infection. Pediatrics $54: 38-42$

15. Manroe B, Rosenfeld C, Weinberg A, Browne R 1977 The differential leukocyte count in the assessment and outcome of early-onset neonatal group B streptococcal disease. J Pediatr 91:632-638

16. Koike K, Nakahata T, Takagi M, Kobayashi T, Ishiguro A, Tsuji K, Naganuma K, Okano A, Akiyama Y, Akabane $\Upsilon 1988$ Synergism of BSF2/interleukin 6 and interleukin 3 on development of multipotential hemopoietic progenitors in serum free culture. J Exp Med 168:879-890

17. Leary A, Ikebuchi K, Hirai Y, Wong G, Yang Y, Clark S, Ogawa M 1988 Synergism between interleukin-6 and interleukin-3 in supporting proliferation of human hematopoietic stem cells: comparison with interleukin-1a. Blood 71:1759-1763

18. Zsebo K, Cohen A. Murdock D, Boone T, Inoue H, Chazin V, Hines D, Souza L 1986 Recombinant human granulocyte colony stimulating factor: molecular and biological characterization. Immunobiology 172:175-184

19. Metcalf D Nicola N 1983 Proliferative effects of purified granulocyte colonystimulating factor (G-CSF) on normal mouse hematopoietic cells. J Cell Physiol 116:198-206

20. Cairo M, Plunkett J, Mauss D, van de Ven C 1990 Seven-day administration of recombinant human granulocyte colony-stimulating factor to newborn rats: modulation of neonatal neutrophilia, myelopoiesis, and group B streptococcus sepsis. Blood 76:1788-1794

21. Asano S, Okano A, Ozawa K, Nakahata T, Ishibashi T, Koike K, Kimura H, Tanioka Y, Shibuya A, Hirano T, Kishimoto T, Takaku F, Akiyama Y 1990 In vivo effects of recombinant human interleukin-6 in primates: stimulated production of platelets. Blood 75:1602-1605

22. Ishibashi T, Kimura H, Shikama Y, Uchida T, Kariyone S, Hirano T, Kishimoto T, Takatsuki F, Akiyama Y 1989 Interleukin-6 is a potent thrombopoietic factor in vivo in mice. Blood 74:1241-1244

23. Takatsuki F Okano A, Suzuki C, Miyasaka Y, Hirano T, Kishimoto T, Ejima D, Akiyama Y 1990 Interleukin 6 perfusion stimulates reconstitution of the immune and hematopoietic systems after 5-fluorouracil treatment. Cancer Res 50:2885-2890

24. Ulich T, del Castillo J, Guo K, Souza L 1989 The hematologic effects of chronic administration of the monokines tumor necrosis factor, interleukin1 , and granulocyte-colony stimulating factor on bone marrow and circulation. Am J Pathol 134:149-159

25. Donahue R, Seehra J, Metzger M, Lefebvre D, Rock B, Carbone S, Nathan D, Garnick M, Sehgal P, Laston D, LaVallie E, McCoy J, Schendel P, Norton C, Turner K, Yang Y, Clark S 1988 Human IL-3 and GM-CSF act synergistically in stimulating hematopoiesis in primates. Science 241:1820-1823

26. Geissler K, Valent P, Mayer P, Liehl E, Hinterberger W, Lechner K, Bettelheim P 1990 Recombinant human interleukin-3 expands the pool of circulating hematopoietic progenitor cells in primates-synergism with recombinant human granulocyte/macrophage colony-stimulating factor. Blood 75:23052310 